DOI: $10.3901 / J M E .2020 .22 .167$

\title{
基于车内噪声的轨道衰减率限值研究*
}

\author{
刘晓龙 ${ }^{1}$ 韩 健 $^{1}$ 徐涆文 $^{1}$ 肖新标 $^{1}$ 朱雷威 $^{2}$ 温泽峰 $^{1}$ \\ (1. 西南交通大学牵引动力国家重点实验室 成都 610031; \\ 2. 中车青岛四方机车车辆股份有限公司 青岛 266000)
}

\begin{abstract}
摘要: 轨道衰减率是反映钢轨动态特性的重要指标, 决定了钢轨的有效声辐射长度, 进一步影响轮轨噪声和车内噪声。我国 地铁轨道结构型式较多, 轨道衰减率也相应地并不统一, 目前我国没有轨道衰减率的相关标准, 现有的国际轨道衰减率标准 并不能与我国车内噪声标准相对应, 因此, 研究轨道衰减率与车内噪声的内在关联, 对基于噪声限值的轨道衰减率控制具有 重要意义。首先建立轨道振动预测模型, 基于现场测试对模型进行验证, 并基于仿真预测分析扣件系统参数对衰减率的影响。 根据有限元-边界元方法和轨道衰减率对轮轨噪声的贡献关系, 建立基于轨道衰减率的轮轨噪声预测模型; 根据线路试验研究, 建立轮轨噪声和车内噪声的传递函数, 从而采取仿真与试验联合的手段, 以轮轨噪声为 “桥梁”, 建立轨道衰减率和车内噪 声的对应关系, 根据车内噪声限值, 量化分析基于车内噪声控制的轨道衰减率限值, 确定较为优化的扣件系统参数。成果可 为基于车内噪声的轨道衰减率控制, 以及扣件系统参数优化设计提供参考。
\end{abstract}

关键词: 轨道衰减率; 轮轨噪声; 车内噪声; 噪声限值; 扣件系统

中图分类号: U211；TB535

\section{Study on the Limiting Value of the Rail Decay Rate Based on Interior Noise}

\section{LIU Xiaolong ${ }^{1}$ HAN Jian $^{1} \quad$ XU Hanwen $^{1} \quad$ XIAO Xinbiao $^{1} \quad$ ZHU Leiwei $^{2} \quad$ WEN Zefeng $^{1}$}

(1. State Key Laboratory of Traction Power, Southwest Jiaotong University, Chengdu 610031;

2. CRRC Qingdao Sifang Co., Ltd., Qingdao 266000)

\begin{abstract}
Rail decay rate is an important indicator to reflect the dynamic properties of the rail, which determines the effective radiating length of the rail, and has an effect on wheel-rail noise and interior noise. There are many types of rail track in Chinese metro lines whose decay rates are significantly different. China has no relevant standards for rail decay rate at present, and the existing international standard cannot correspond with the Chinese railway noise standard. So, researching the relationship between the interior noise and rail decay rate is significant in controlling the rail decay rate based on interior noise limit. Rail vibration prediction model, which is verified by the field test results, is established to analyze the effect of the fastener parameters on rail decay rate. The wheel/rail noise simulation model is established based on the rail decay rate according to the FEM-BEM and the relationship between the decay rate and wheel/rail noise. Then the transfer relationship between wheel/rail noise and interior noise is obtained according to the field test. Simulation combined with field test method is used in this research to establish the correspondence between the track attenuation rate and the interior noise, in which the wheel/rail noise plays a role of a bridge. Next, the decay rate limit based on interior noise is quantified according to the interior noise. Finally, fastener parameters according to the decay rate character is optimized. The related research results provide a reference on rail decay rate control based on interior noise and fastener parameters optimization.
\end{abstract}

Key words: rail decay rate; wheel-rail noise; interior noise; sound limits; fastener system

0 前言

钢轨是轮轨噪声中主要的噪声源之一 ${ }^{[1-2]}$, 在轮

* 国家重点研发计划(2017YFB1201103-08)和四川省科技计划(19GJHZ0062) 资助项目。20191109 收到初稿, 20200528 收到修改稿
轨噪声贡献频谱中 (图 1$)^{[3]}$, 钢轨声辐射在 $400 \sim$ $2000 \mathrm{~Hz}$ 倍频程范围内占据了主导地位。我国地 铁轨道结构类型多样, 线路环境复杂, 有效地研 究轨道的固有特性及其与噪声的关系, 对地铁噪 声控制具有重要意义。轨道振动沿着钢轨纵向的 衰减率作为轨道的一个固有特性, 用以表征钢轨 
振动沿钢轨纵向衰减的快慢程度。较高的衰减率 使得钢轨振动能量能够快速衰减, 钢轨有效声辐 射长度更短, 从而辐射更小的钢轨噪声; 反之, 钢轨辐射较大噪声。THOMPSON 等 ${ }^{[4-6]}$ 常常将轨 道振动衰减率的测试结果用于对轨道声辐射进行 预测, 以及对轮轨噪声预测模型的验证。JONES 等 ${ }^{[7]}$ 分别利用现场测试和仿真计算得到的衰减率 去预测轮轨噪声, 并且与现场测试得到的轮轨噪 声进行了对比。 $\mathrm{WU}^{[8]}$ 建立了离散支撑轨道分别与 离散分布、连续分布的钢轨阻尼器的联合模型, 研究了钢轨阻尼器对钢轨振动衰减率与滚动噪声 的影响。孙晓静等 ${ }^{[9]}$ 通过现场锤击试验, 测试了 某地铁轨道衰减率, 并初步分析了轨道衰减率与 钢轨波磨以及扣件弹条断裂等问题之间的关系, 研究发现, 过小的轨道衰减率导致轮轨剧烈振动 不易衰减, 引起钢轨波磨快速发展; ZHANG 等 ${ }^{[10]}$ 通过试验研究了钢轨阻尼器对钢轨波磨的控制, 分析了钢轨阻尼、轨道衰减率以及钢轨波磨之间 的影响关系。

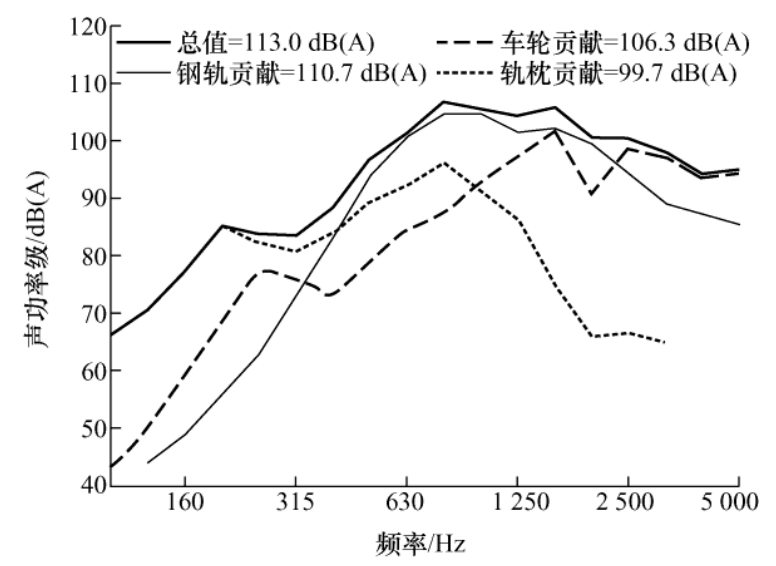

图 1 轮轨噪声成分频谱特性

以上研究表明, 轨道衰减率在一定程度上影响 着轨道声辐射以及轨道系统的破坏, 以往对轨道衰 减率的影响因素以及其对轮轨振动噪声影响开展了 较多的研究, 但随着我国地铁轨道型式的多样化, 轨道支承刚度大小不一, 在减振的同时, 往往忽略 了车内噪声问题, 通过轨道衰减率实现对车内噪声 控制的相关研究鲜见报道。本文基于仿真与试验联 合的手段, 以轮轨噪声为 “桥梁”, 建立轨道衰减率 和车内噪声的对应关系, 判断不同等级轨道衰减率 下车内噪声是否满足噪声限值要求, 量化分析基于 车内噪声控制的轨道衰减率限值, 进一步根据不同 扣件参数对轨道衰减率的影响规律, 确定较为优化 的扣件系统参数。

\section{1 轨道振动衰减率的试验研究}

\section{1 轨道振动衰减率}

在轨道衰减率的理论定义中, 通常需要考虑垂 向振动与横向振动的弯曲波, 对钢轨振动进行波数 分解。垂向、横向振动弯曲波(包括近场波与传播波) 沿着钢轨纵向以指数规律衰减, 如式(1)所示

$$
R(x) \approx R(0) \exp \left(-k_{i} x\right)
$$

式中, $R(x)$ 表示钢轨垂向与横向弯曲波沿钢轨纵向 的振动响应; $R(0)$ 表示激励点(轮轨接触点)钢轨振动 响应; $k_{i}$ 为复波数的虚部, 表示衰减系数, 将该系 数转换为衰减率的形式, 则有

$$
D R=20 \lg \exp k_{i}=8.686 k_{i}
$$

式中, $D R$ 为钢轨振动衰减率。

\section{2 衰减率试验研究方法}

在工程实践中, 轨道振动衰减率的测算, 通常 是获取钢轨振动的整体响应(各种形式的波的叠 加), 而无需进行波数分解。轨道衰减率的测试分为 短轨法与长轨法。短轨法适用于阻尼钢轨的实验室 测试, 详见文献[11-12]。长轨法 ${ }^{[13]}$ 主要运用于实际 运营线路, 需要在沿钢轨长度方向上, 分别测试一 系列钢轨垂向激励下的垂向频率响应函数, 根据振 幅衰减法进行轨道衰减率估计。为此需要使用力锤 对钢轨进行激励, 为提高试验效率, 基于互易性原 理, 采取移动力锤法进行试验, 试验激励点与响应 点如图 2 所示。图 $2 \mathrm{a}$ 中, 编号 1 表示力锤垂向激励 位置, 编号 2 为可选的垂向响应位置。图 $2 b$ 中的激 励点分为近场点与远场点, 由于近场波的存在, 近 场点的测试精度对于低频段的高衰减率非常重要, 对于远场点, 并不需要对每一跨的振动响应进行测 试, 相应的锤击点随着距离的增加而更稀疏地分布。

将得到的一系列窄带频率响应函数转换为 $1 / 3$ 倍频程谱, 如果 $A(x)$ 表示距离激励点 $x$ 处钢轨的某 $1 / 3$ 倍频程带宽内的频率响应函数幅值, 则钢轨辐 射振动能量可以表达为幅值的平方沿钢轨纵向的 积分

$$
\int_{0}^{\infty}|A(x)|^{2} \mathrm{~d} x=|A(0)|^{2} \int_{0}^{\infty} \exp \left(-2 k_{i} x\right) \mathrm{d} x=|A(0)|^{2} \frac{1}{2 k_{i}}
$$

若采用离散点锤击测试的钢轨轨头频响函数, 则式(3)可表示为

$$
\int_{0}^{\infty} \frac{|A(x)|^{2}}{|A(0)|^{2}} \mathrm{~d} x=\frac{1}{2 k_{i}} \approx \sum_{n=0}^{n_{\max }} \frac{\left|A\left(x_{n}\right)\right|^{2}}{|A(0)|^{2}} \Delta x_{n}
$$




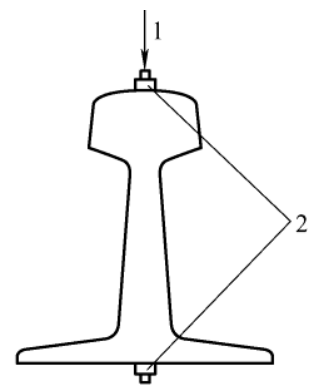

(a) 响应点分布

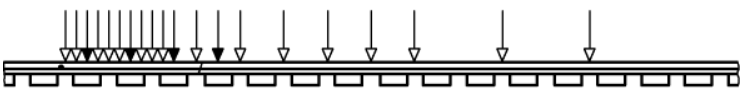

$\begin{array}{llllllllllllllll}0 & 1 & 2 & 3 & 4 & 5 & 6 & 7 & 8 & 9 & 10 & 11 & 12 & 13 & 14 & 15\end{array}$

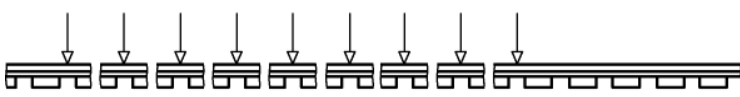

$\begin{array}{lllllllllllll}15 & 16 & 20 & 24 & 30 & 36 & 42 & 48 & 54 & 66 & 67 & 68 & (\ldots)\end{array}$

(b) 激励点分布

图 2 长轨法衰减率测试示意图

结合式(2)、(4), 可得衰减率表达式

$$
D R \approx \frac{4.343}{\sum_{n=0}^{n_{\max }} \frac{\left|A\left(x_{n}\right)\right|^{2}}{|A(0)|^{2}} \Delta x_{n}}
$$

式中, $A(0)$ 表示第一个激励点每个 $1 / 3$ 倍频程中心频 率频响函数幅值; $A\left(x_{n}\right)$ 表示第 $n$ 个锤击点在每个 $1 / 3$ 倍频程中心频率频响函数幅值; $\Delta x_{n}$ 表示第 $n$ 个锤 击点与参考点之间的距离。

\section{3 试验结果分析}

根据以上介绍的衰减率测试与处理方法, 本文 分别对某地铁线路上 DTVI2 型扣件整体道床轨道 和 DTVII2 型减振扣件整体道床轨道进行了衰减率 测试, 如图 3 所示, 试验结果如图 4 所示。

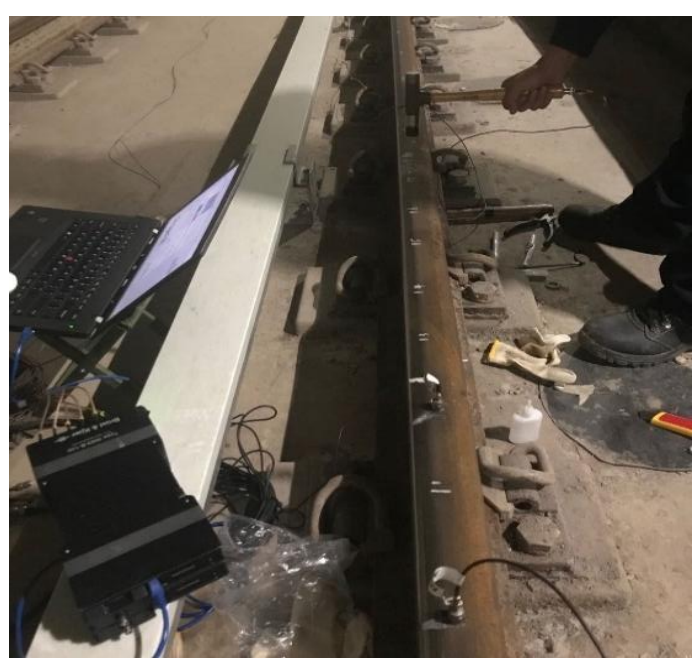

图 3 轨道衰减率测试

图 4 中, 两种轨道的衰减率随频率变化具有较 为一致的变化规律：低频段 $(200 \mathrm{~Hz}$ 以下)出现的衰 减率峰值, 是因为近场波的存在, 以及轨枕和轨道
板的动力吸振作用, 使得钢轨振动几乎不衰减地传 递到轨枕、轨道板以及地面; 随着频率的升高, 自 由传播的波出现后，钢轨和轨枕的振动不再同步， 此时衰减率出现急剧下降的趋势; 高频处衰减率再 次上升, 主要是因为钢轨出现截面变形, 包括轨脚 的摆动等, 增加了轨垫板的阻尼效果。总体上, DTVI2 型扣件轨道的振动衰减率要高于 DTVII2 减 振扣件轨道, 在 $600 \mathrm{~Hz}$ 倍频程带以下较为明显; 高于 $600 \mathrm{~Hz}$ 时, 两者衰减率趋于一致。两种轨道 之间主要存在扣件刚度、阻尼的差异。

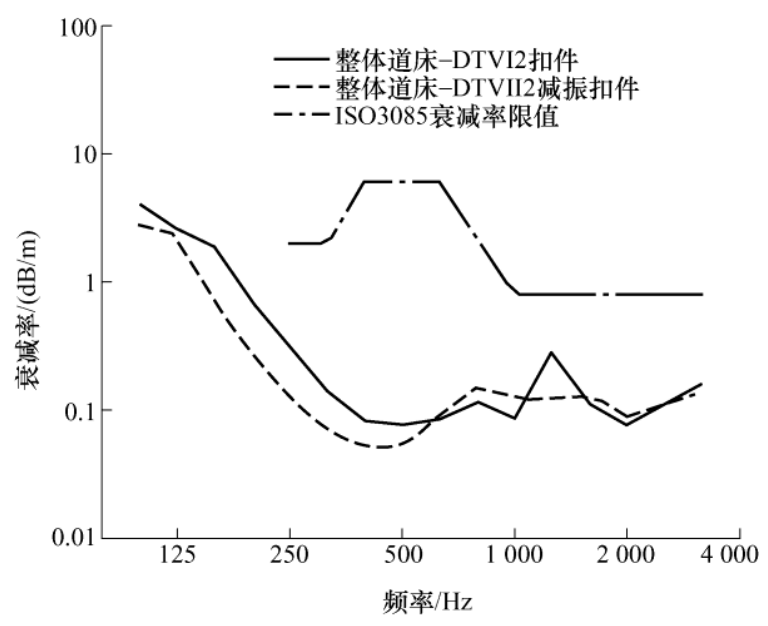

图 4 轨道衰减率测试结果

\section{2 轨道振动衰减率的影响因素研究}

由第 1.3 节分析可知, 扣件系统的参数, 会显著 影响轨道的振动衰减率。本节为了研究扣件参数对轨 道衰减率的影响, 分别用铁木辛柯梁理论与有限元方 法对轨道进行建模, 计算钢轨振动响应, 并确定轨道 振动衰减率, 计算中扣件阻尼为 $1 \times 10^{4} \mathrm{~N} \cdot \mathrm{s} / \mathrm{m}$, 刚度 为 $20 \times 10^{6} \mathrm{~N} / \mathrm{m}$ 。图 5 给出了两种理论方法计算结果 与试验结果的对比。

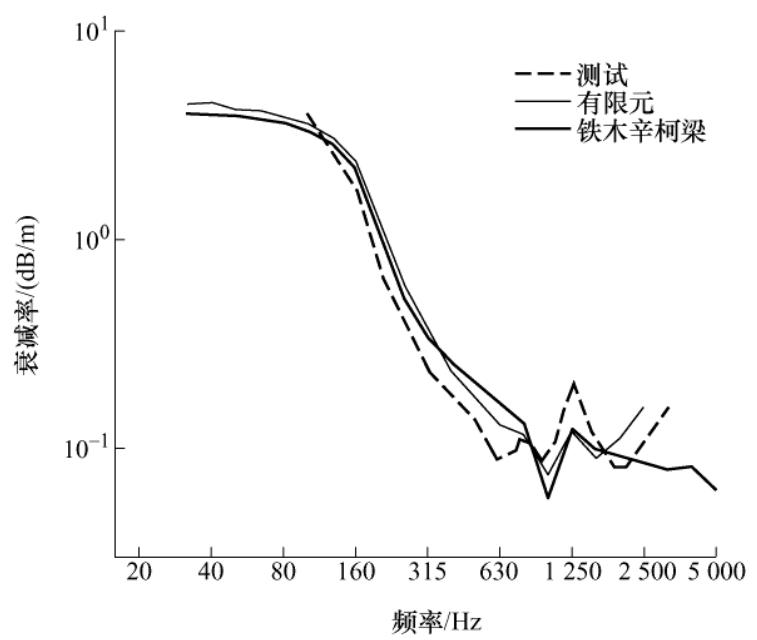

图 5 衰减率计算结果对比 
由图 5 可知, 在 $2500 \mathrm{~Hz}$ 倍频程带以下, 两种 理论方法计算结果和测试结果吻合得比较好, 高于 $2500 \mathrm{~Hz}$, 有限元计算结果与测试结果具有相同的 变化趋势, 而铁木辛柯梁理论计算结果出现了较大 的差异。出现这种情况主要是因为在高频段钢轨截 面发生变形, 而铁木辛柯梁只考虑了梁的弯曲与截 面转动。由此可见, 铁木辛柯梁仍可以在 $2500 \mathrm{~Hz}$ 以下保证较为准确的轨道衰减率预测, 考虑到有限 元方法计算效率较低, 当网格必须达到 $2500 \mathrm{~Hz}$ 以 上计算要求时, 计算时间约为梁模型的十倍以上; 结合本文关注的噪声问题和轨道衰减率均未超过 $2500 \mathrm{~Hz}$, 因此本文采用铁木辛柯梁模拟钢轨来计 算轨道衰减率。

扣件刚度、阻尼以及扣件间距是扣件系统中 几项重要的参数, 对轨道的振动特性均有影响, 以下分别从这三个方面对轨道衰减率的影响进行 研究。

\section{1 扣件系统刚度}

扣件系统刚度决定了钢轨与轨枕、轨道板的耦 合振动特性, 影响着钢轨振动向轨枕、轨道板的传 递。振动的垂向传递能力越强, 残留于钢轨的振动 能量越小, 钢轨的振动衰减率越大。本节在常见的 扣件刚度范围 $\left(10 \times 10^{6} \sim 100 \times 10^{6} \mathrm{~N} / \mathrm{m}\right)$ 内, 设置了 $20 \times 10^{6} \mathrm{~N} / \mathrm{m} 、 40 \times 10^{6} \mathrm{~N} / \mathrm{m} 、 60 \times 10^{6} \mathrm{~N} / \mathrm{m}$ 以及 $80 \times$ $10^{6} \mathrm{~N} / \mathrm{m}$ 四个刚度等级。轨枕间距为 $0.6 \mathrm{~m}$, 扣件 阻尼为 $1 \times 10^{4} \mathrm{~N} \cdot \mathrm{s} / \mathrm{m}$ 。

图 $6 \mathrm{a}$ 给出了四种刚度下钢轨振动原点加速度 导纳, 曲线中存在两个显著峰值, 第一个高阻尼峰 值约为 $200 \mathrm{~Hz}$, 该峰值频率是与扣件刚度有关的钢 轨共振频率; 第二个峰值出现在 $1000 \mathrm{~Hz}$ 左右, 为 轨道的 pinned-pinned 共振模态。随着扣件刚度的增 加, 第一个峰值频率逐渐增加, 钢轨振动幅值也有 所增加; 而刚度的改变对 $400 \mathrm{~Hz}$ 以上频率的振动 基本没有影响。

图 $6 \mathrm{~b}$ 为四种刚度下钢轨的振动衰减率结果, $400 \mathrm{~Hz}$ 倍频程以下的振动衰减率随着扣件刚度的 增加有显著的提高, 刚度每提高 $20 \times 10^{6} \mathrm{~N} / \mathrm{m}$, 频段 内衰减率平均提高约 $0.8 \mathrm{~dB} / \mathrm{m}$ 。对 $400 \mathrm{~Hz}$ 倍频程 以上的振动衰减率基本没有影响。综上, 扣件系统 刚度的增加, 使得钢轨与轨下结构耦合作用增强, 虽然原点导纳振幅有一定增加, 但钢轨振动能量更 容易向下传递, 使得残留于钢轨上的整体振动能量 减小, 即钢轨振动衰减率增加。因此, 在轨道设计 过程, 需要提高轨道中低频段的轨道衰减率, 可以 从增加扣件系统的刚度考虑。

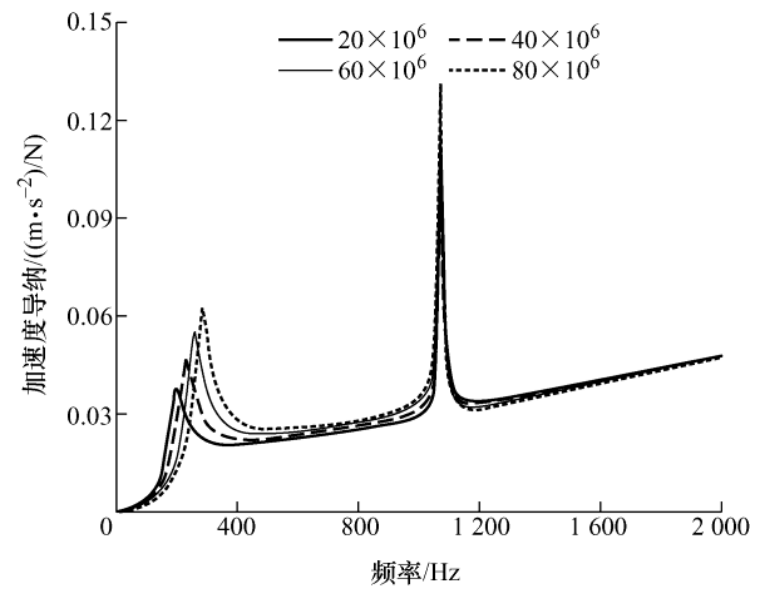

(a) 钢轨加速度导纳

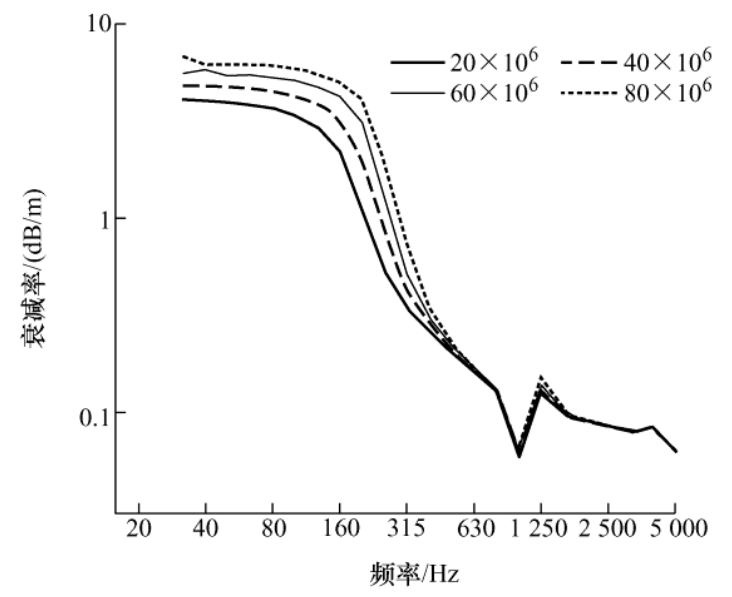

(b) 轨道振动衰减率

图 6 扣件刚度的影响

\section{2 扣件系统阻尼}

为了研究扣件系统阻尼对钢轨振动特性的影响, 本节在常见的扣件阻尼范围内设置了 $1 \times 10^{4} \mathrm{~N} \cdot \mathrm{s} / \mathrm{m}$ 、 $2 \times 10^{4} \mathrm{~N} \cdot \mathrm{s} / \mathrm{m} 、 3 \times 10^{4} \mathrm{~N} \cdot \mathrm{s} / \mathrm{m}$ 以及 $4 \times 10^{4} \mathrm{~N} \cdot \mathrm{s} / \mathrm{m}$ 四个阻尼等级。扣件刚度为 $20 \times 10^{6} \mathrm{~N} / \mathrm{m}$, 轨枕间距 为 $0.6 \mathrm{~m}$ 。

图 $7 \mathrm{a}$ 给出了不同阻尼等级下的钢轨振动原点 加速度导纳曲线, 阻尼的增加对钢轨振动的频率特 性影响很小, 主要影响钢轨振动幅值, 使得钢轨在 显著峰值处更加平缓。

图 $7 \mathrm{~b}$ 为四种阻尼下钢轨的振动衰减率结果, 随 着阻尼每增加 $1 \times 10^{4} \mathrm{~N} \cdot \mathrm{s} / \mathrm{m}$, 轨道振动衰减率在全 频段内都有所增加, 在 $125 \mathrm{~Hz}$ 倍频程带以下, 衰 减率提高较小, 平均约为 $0.02 \mathrm{~dB} / \mathrm{m}$, 而在高于 $125 \mathrm{~Hz}$ 倍频程带处, 衰减率增加较为显著, 平均增 加约为 $0.18 \mathrm{~dB} / \mathrm{m}$, 但是在钢轨 pinned-pinned 共振 频率处, 钢轨振动衰减率并没有得到提高。因此, 在轨道设计过程, 需要提高中高频段(pinned-pinned 频率峰值除外)的轨道衰减率, 可以从增加扣件系统 的阻尼考虑。 


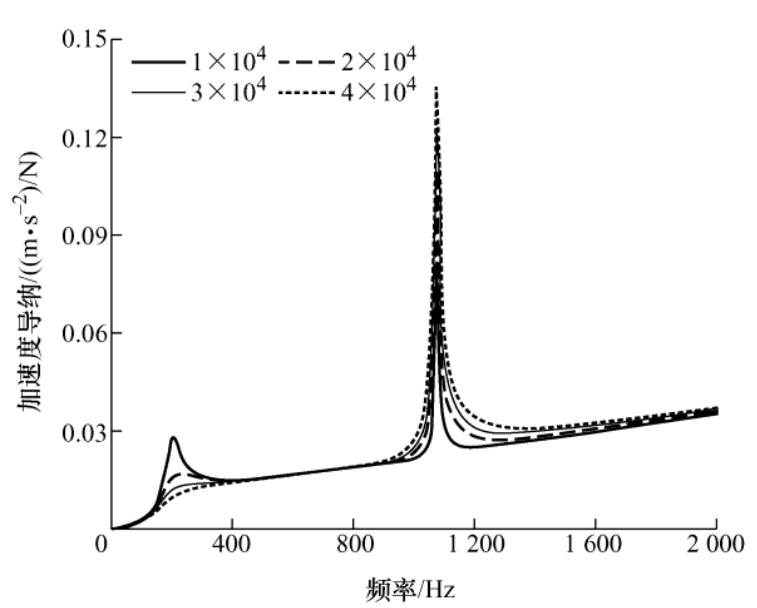

(a) 钢轨加速度导纳

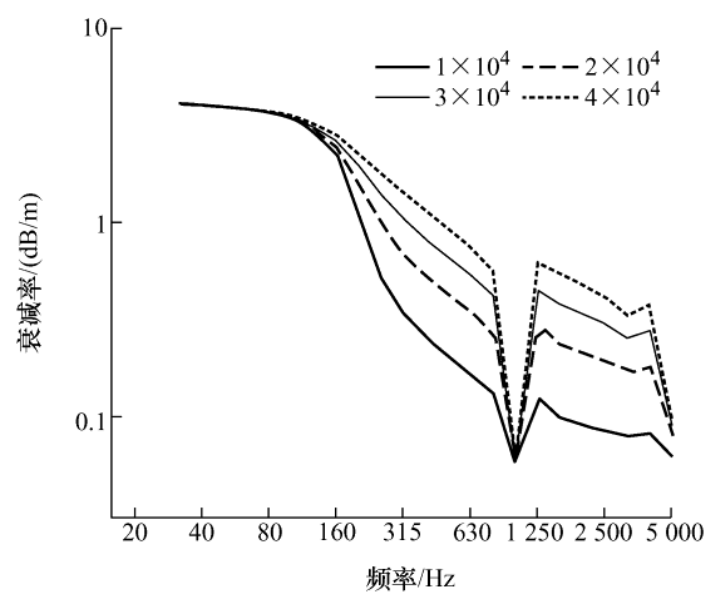

(b) 轨道振动衰减率

图 7 扣件阻尼的影响

\section{3 扣件系统间距}

为了研究扣件系统间距对钢轨振动特性的影 响, 本节设置了 $0.55 \mathrm{~m} 、 0.6 \mathrm{~m} 、 0.65 \mathrm{~m}$ 以及 $0.7 \mathrm{~m}$ 四个等级的扣件间距。扣件刚度为 $20 \times 10^{6} \mathrm{~N} / \mathrm{m}$, 扣 件阻尼为 $1 \times 10^{4} \mathrm{~N} \cdot \mathrm{s} / \mathrm{m}$ 。

图 $8 \mathrm{a}$ 给出了四种扣件间距钢轨振动原点加速 度导纳, 曲线中的第一个与轨下结构相关的峰值未 随扣件间距的增加而发生改变, 而钢轨 pinnedpinned 共振频率随扣件间距的增加而向低频移动。

图 $8 \mathrm{~b}$ 为四种扣件间距下轨道的振动衰减率结 果。扣件间距的改变主要对低频以及 pinnedpinned 共振频率产生影响, 对低于 $160 \mathrm{~Hz}$ 倍频程 带的衰减率, 扣件间距减小会适当减小轨道衰减 率, 但对于 pinned-pinned 频率处的轨道衰减率具 有增加的作用, 在轨道设计过程, 需要提高轨道 pinned-pinned 频率附近的轨道衰减率, 可以从适 当减小扣件系统的间距考虑, 由于扣件间距减小 导致的低频衰减率减小, 可通过刚度来综合 调节。

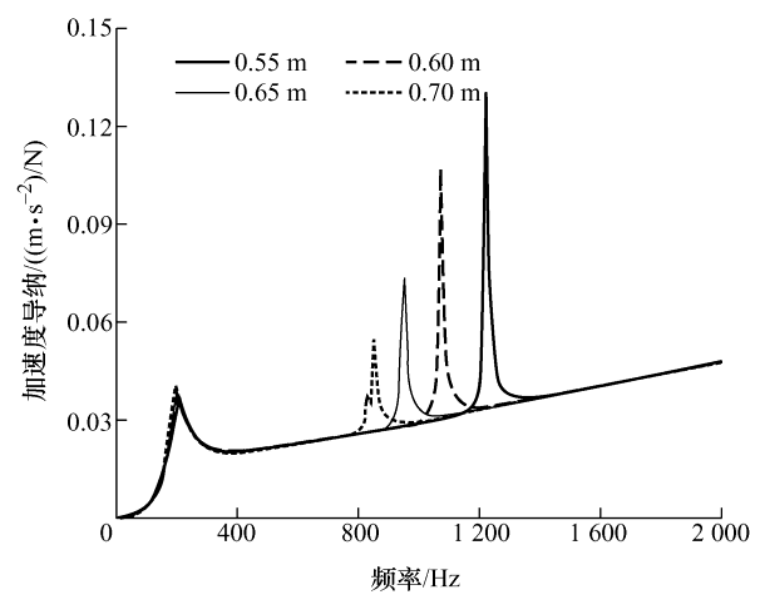

(a) 钢轨加速度导纳

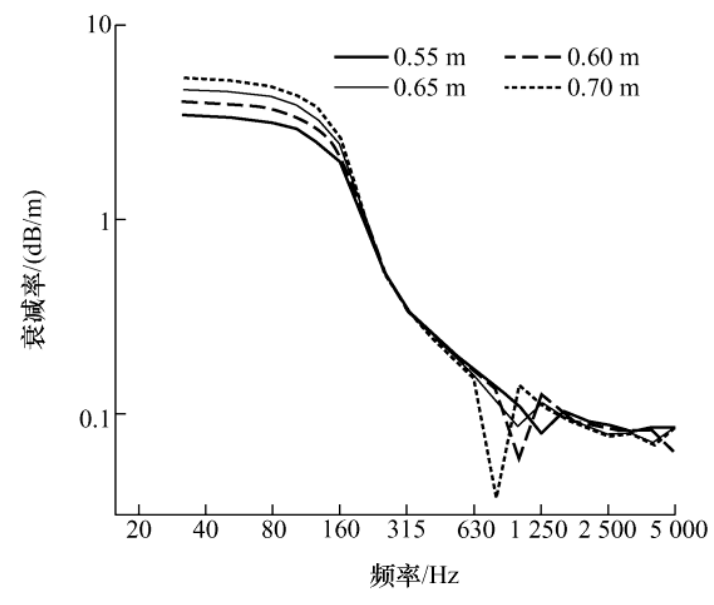

(b) 轨道振动衰减率

图 8 扣件间距的影响

3 基于车内噪声控制的轨道衰减率 限值分析

\section{1 衰减率与车内噪声关系建立}

振动结构辐射的声功率 $W$ 一般可用式(6)表示

$$
W=\frac{1}{2} \rho_{0} c_{0} \sigma P \int_{-\infty}^{\infty}|v(x)|^{2} \mathrm{~d} x
$$

式中, $v(x)$ 是在 $x$ 处的速度幅值; $\rho_{0} c_{0}$ 是空气中的 声特性阻抗, 一般取 $20{ }^{\circ} \mathrm{C}$ 时的值, 即 $\rho_{0} c_{0}=$ $415 \mathrm{~N} \cdot \mathrm{s} / \mathrm{m}^{3} ; \sigma$ 是与频率相关的声辐射效率; $P$ 为 一个截面的周长。将钢轨简化为简支梁模型, 并考 虑 $x=0$ 处的对称性, 可得式(7), 由于隐失波随 $x$ 迅速衰减，积分后对结果影响很小，可进一步得到 式(8)

$$
\begin{gathered}
W=\rho_{0} c_{0} \sigma P \frac{|v(0)|^{2}}{2} \int_{0}^{\infty}|\exp (-i k x)-i \exp (-k x)|^{2} \mathrm{~d} x \\
W \approx \frac{1}{2} \rho_{0} c_{0} \sigma P|v(0)|^{2} \int_{0}^{\infty}|\exp (-i k x)|^{2} \mathrm{~d} x
\end{gathered}
$$

式中, $v(0)$ 为轮轨作用点钢轨振动速度幅值, 对于 
一个复波数 $k=k_{r}+i k_{i}$, 式(8)中的积分项可简化为

$$
\int_{0}^{\infty}|\exp (-i k x)|^{2} \mathrm{~d} x=\int_{0}^{\infty} \exp \left(2 k_{i} x\right) \mathrm{d} x=\frac{-1}{2 k_{i}}
$$

将式(9)代入式(8)得到

$$
W \approx \frac{\rho_{0} c_{0} \sigma P|v(0)|^{2}}{-4 k_{i}}
$$

将式(2)代入式(10)可得到用轨道衰减率表示的 钢轨声功率

$$
W \approx \frac{4.343 \rho_{0} c_{0} \sigma P|v(0)|^{2}}{2 D R}
$$

由以上推导可知, 在获取了轨道衰减率之后, 通过式(11)可近似计算钢轨辐射声功率, 再由有限 元-边界元方法, 计算轮轨力作用下车轮声辐射, 进 一步得到轮轨噪声 ${ }^{[14]}$ 。首先在单位力激励下分别计 算出车轮导纳 $\alpha_{W}$ 、钢轨导纳 $\alpha_{R}$ 与接触导纳 $\alpha_{C}$, 并根 据实测轮轨粗楉度, 考虑接触滤波计算出轮轨联合 粗粘度 $r$, 由式(12)求出轮轨相互作用力, 将该轮轨 力作为激励输入, 求得车轮振动响应, 再由三维声 学积分方程 ${ }^{[15]}$ (式(13)) 计算出车轮噪声。车轮有限元 模型中, 采用普通直辐板车轮, 直径 $840 \mathrm{~mm}$, 由 solid45 实体单元进行离散。材料弹性模量为 $2.1 \times 10^{11} \mathrm{~Pa}$, 泊松比为 0.3 , 密度为 $1850 \mathrm{~kg} / \mathrm{m}^{3}$ 。

$$
\begin{gathered}
F=r /\left(\alpha_{R}+\alpha_{W}+\alpha_{C}\right) \\
\bar{p}(x, y, z)=-\int_{S}\left(\bar{p}\left(x^{\prime}, y^{\prime}, z^{\prime}\right) \frac{\partial G(R)}{\partial n}+\right. \\
\left.i \rho \omega G(R) v_{n}\left(x^{\prime}, y^{\prime}, \mathrm{z}^{\prime}\right)\right) \mathrm{d} S
\end{gathered}
$$

式中, $\bar{p}(x, y, z)$ 表示空间某一点的声压幅值; $v_{n}\left(x^{\prime}, y^{\prime}, \mathrm{z}^{\prime}\right)$ 为空气质点沿法向的振动速度; $G(R)$ 为 格林函数, 运用三维边界元方法求解式(13)。

地铁列车轮轨噪声是主要声源, 而且列车在隧 道内, 运行环境近似为混响场, 在此前提下, 可根 据轮轨噪声与列车噪声平均传递关系, 求得车内噪 声。列车噪声平均传递关系, 通过现场试验确定。 至此, 以轮轨噪声作为 “桥梁”, 即可建立轨道衰减 率与车内噪声的联系。大量的现场测试发现, 目前 绝大多数地铁线路中, 出于对轨道的减振设计, 使 得轨道的衰减率均处于较低的水平, 远低于 ISO 3095 中的轨道衰减率限值 ${ }^{[16]}$, 如图 4 所示。在轮轨 粗鉌度不变的情况下, 以轨道衰减率作为输入变量, 计算车内噪声, 判断是否满足车内噪声限值要求; 如不满足，增加相应频段内的轨道衰减率进入下一 个循环, 直到满足噪声限值, 确定基于车内噪声的 轨道衰减率限值。计算中钢轨声辐射效率 $\sigma$ 如图 9 所示。

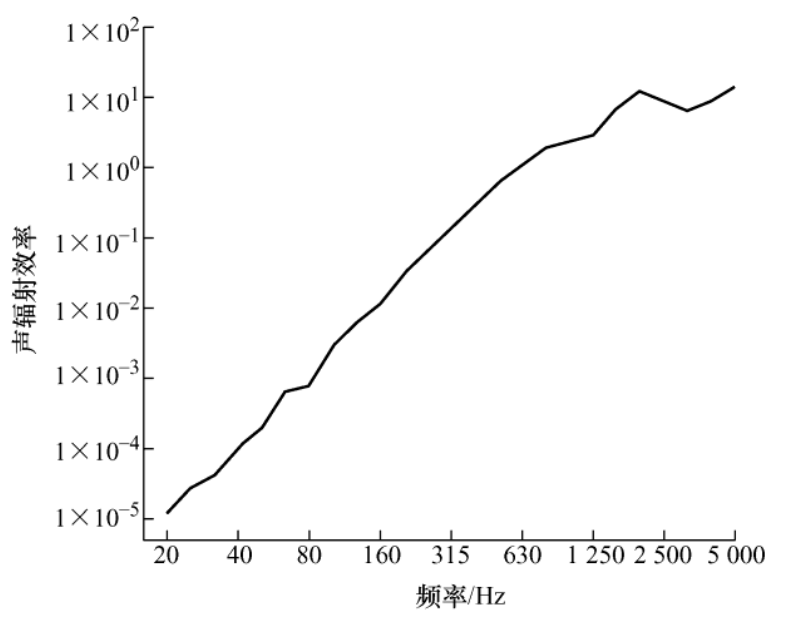

图 9 钢轨声辐射效率

\section{2 基于车内噪声控制的轨道衰减率限值分析}

现行的相关国际标准，对轨道衰减率的限值要 求较高, 绝大多数轨道衰减率均远低于标准限值; 且不能与我国轮轨噪声和车内噪声标准相对应。因 此, 研究车内噪声与轨道衰减率的关系, 建立基于 车内噪声的轨道衰减率限值具有重要意义。

图 5 中验证后的有限元模型能准确地反映某种 地铁常用轨道类型的衰减率，以该有限元计算得到 的衰减率作为基准, 以 $\mathrm{A}$ 级车轮粗粘度谱和 $\mathrm{C}$ 级 钢轨粗粘度谱 ${ }^{[17]}$ 作为激励, 设列车运行速度为 $70 \mathrm{~km} / \mathrm{h}$, 在某地铁列车司机室噪声传递关系基础 上, 得到司机室车内噪声频谱如图 10 所示, 此时车 内噪声总值为 $83.36 \mathrm{~dB}(\mathrm{~A})$, 超出地铁司机室噪声限 值 ${ }^{[18]} 3.36 \mathrm{~dB}(\mathrm{~A})$ 。

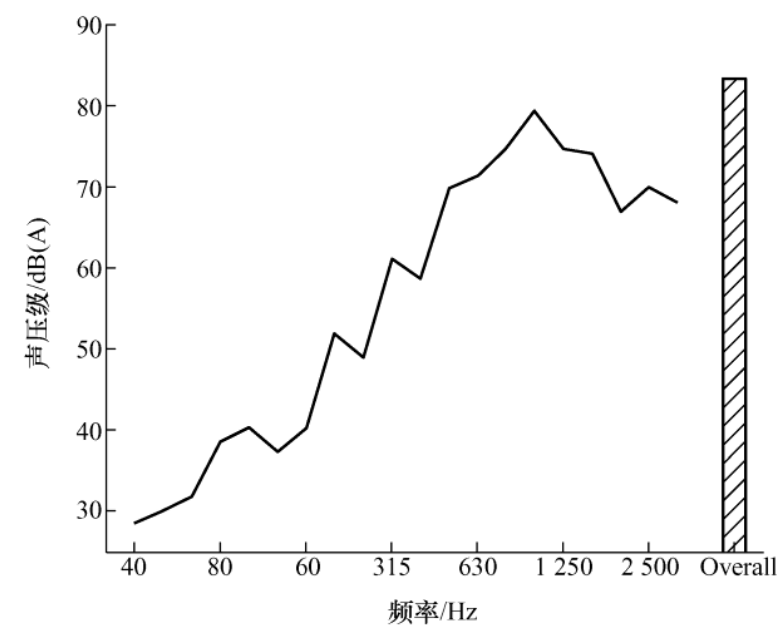

图 10 车内噪声

根据衰减率的变化特征(低频衰减率大，高频衰 减率小), 将上述轨道衰减率分为低频段(31.5 $160 \mathrm{~Hz}$ )和高频段 $(200 \sim 2500 \mathrm{~Hz}$ )两部分, 分别研究 车内噪声总值的影响。图 11 为车内噪声总值随衰减率 变化之间的关系。当 $31.5 \sim 160 \mathrm{~Hz}$ 频率段内衰减率增 
加 $20 \mathrm{~dB} / \mathrm{m}$ 时, 车内噪声总值仅降低了约 $0.1 \mathrm{~dB}(\mathrm{~A})$; 而当 200 2 $500 \mathrm{~Hz}$ 频率段内衰减率增加 $0.1 \mathrm{~dB} / \mathrm{m}$, 车内噪声降低约 $2.2 \mathrm{~dB}(\mathrm{~A})$, 当衰减率提高 $0.3 \mathrm{~dB} / \mathrm{m}$ 时, 车内噪声降低约 $3.9 \mathrm{~dB}(\mathrm{~A})$, 此时车内噪声总值 为 $79.4 \mathrm{~dB}(\mathrm{~A})$, 已低于司机室车内噪声限值。随着 衰减率的进一步提高, 车内噪声将进一步降低, 但 是降低率逐渐减小。

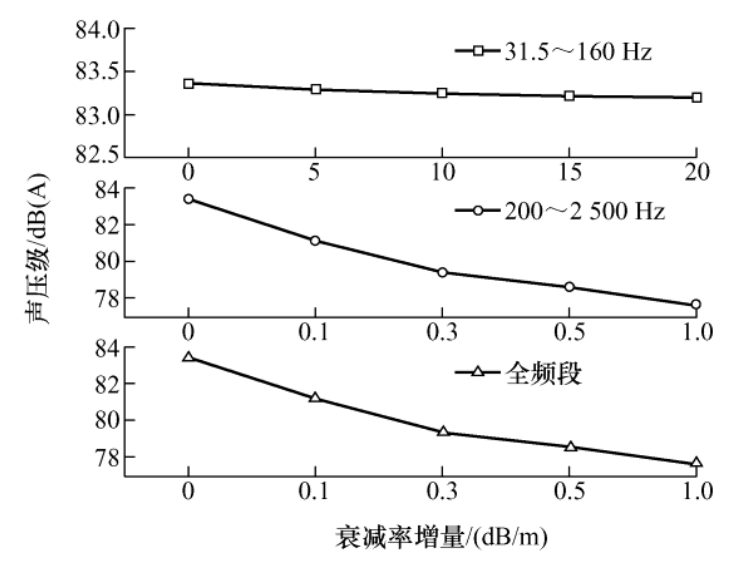

图 11 车内噪声总值

以上给出了各频段衰减率与车内噪声总值的对 应关系, 接下来, 通过增加全频段内的衰减率, 研 究衰减率与噪声的频谱特性, 如图 12 所示。图 13 分别给出了不同水平轨道衰减率所对应的车内噪声 频谱。随着轨道衰减率的增加, 低频段钢轨声辐射 与车内噪声几乎没有变化, 而在高频段, 车内噪声 显著减小, 从而车内噪声总值显著降低。

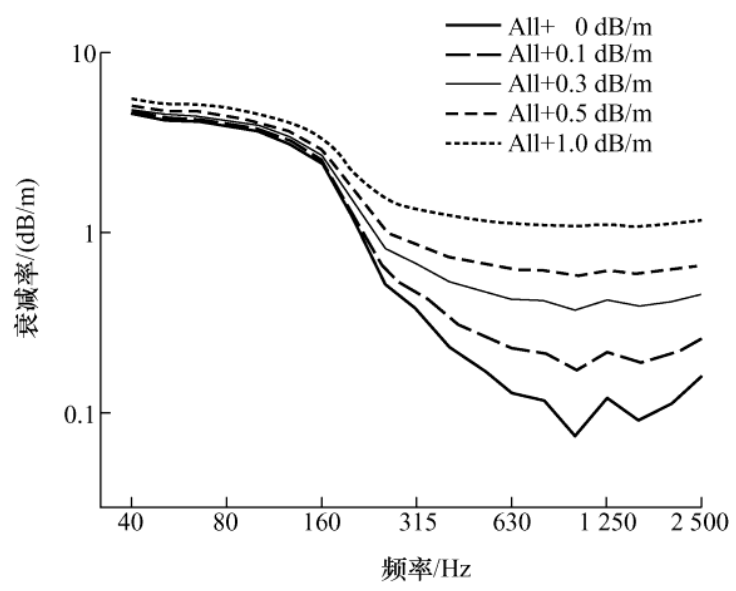

图 12 全频段衰减率调查

根据以上分析可知, 低频段的轨道衰减率对车 内噪声几乎没有影响, 主要是因为, 在低频时钢轨 声辐射效率非常小, 衰减率的变动对钢轨声辐射影 响很小; 而高频段钢轨声辐射在轮轨噪声中占据主 导地位, 车内噪声受到该频段轨道衰减率的影响较 大。由此, 得出基于车内噪声控制的轨道衰减率限

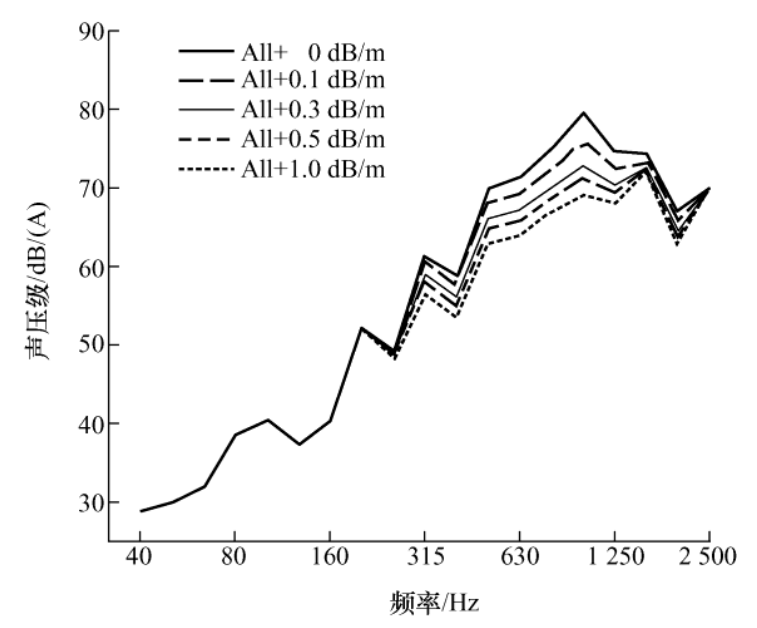

图 13 全频段衰减率与车内噪声

值, 如图 14 所示, 该限值与现有 ISO 3095 标准中 的衰减率限值相比, 放宽了对轨道衰减率的要求, 但同时又能够满足车内噪声限值的要求, 这对于 轨道设计与车内噪声控制具有重要的指导意义。 结合第 2 节衰减率影响因素研究结果, 从降低车 内噪声的角度, 最行之有效的方法是增加扣件系 统的阻尼, 如采用高阻尼材料的轨下垫片等, 使 得扣件系统阻尼为 $3 \times 10^{4} \mathrm{~N} \cdot \mathrm{s} / \mathrm{m}$ 左右, 从而达到 衰减率的控制要求。而增加扣件刚度和扣件系统 间距, 虽然能提高低频轨道衰减率, 但对车内噪 声几乎没有影响。

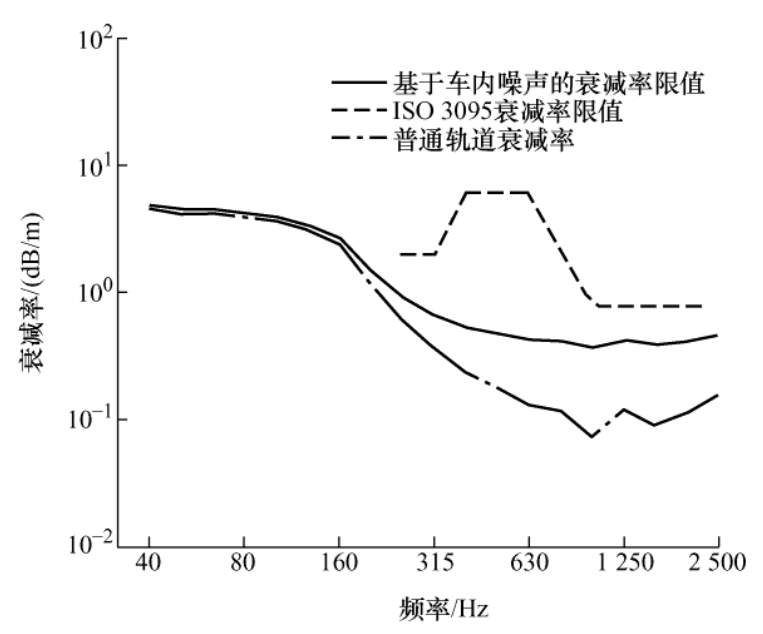

图 14 基于车内噪声的轨道衰减率限值

\section{4 结论}

本文首先介绍了轨道衰减率的测试和计算方 法, 以及随频率的变化趋势; 然后, 基于铁木辛柯 梁理论, 建立轨道振动预测模型, 分析了扣件刚度、 阻尼以及扣件间距对轨道衰减率的影响; 再结合现 场试验结果, 建立了列车噪声平均传递关系, 并基 
于轮轨相互作用理论、轨道衰减率与钢轨声辐射之 间的关系, 以及有限元-边界元方法, 计算了车内噪 声与轨道衰减率之间的量化关系; 最后基于车内噪 声限值, 提出了轨道衰减率限值要求。具体研究结 论如下。

（1）扣件刚度每增加 $20 \times 10^{6} \mathrm{~N} / \mathrm{m}, 400 \mathrm{~Hz}$ 倍频 程带以下衰减率平均提高约 $0.8 \mathrm{~dB} / \mathrm{m}$, 对 $400 \mathrm{~Hz}$ 倍频程带以上的振动衰减率基本没有影响; 扣件阻 尼每增加 $1 \times 10^{4} \mathrm{~N} \cdot \mathrm{s} / \mathrm{m}$, 在高于 $125 \mathrm{~Hz}$ 倍频程带 处, 衰减率平均增加约为 $0.18 \mathrm{~dB} / \mathrm{m}$, 对低于 $125 \mathrm{~Hz}$ 的衰减率影响较小; 扣件间距每增加 $0.05 \mathrm{~m}$, 低于 $160 \mathrm{~Hz}$ 倍频程带的衰减率平均增加约 $0.5 \mathrm{~dB} / \mathrm{m}$, 对高于 $160 \mathrm{~Hz}$ 的衰减率几乎没有影响。

(2) 在扣件参数为阻尼 $1 \times 10^{4} \mathrm{~N} \cdot \mathrm{s} / \mathrm{m}$, 刚度 $20 \times 10^{6} \mathrm{kN} / \mathrm{mm}, \mathrm{A} / \mathrm{C}$ 级轮/轨粗粘度激励下, 该列 车司机室内噪声超标约 $3.36 \mathrm{~dB}(\mathrm{~A})$ 。低频轨道衰减 率对车内噪声的影响很小, 当 $31.5 \sim 160 \mathrm{~Hz}$ 频率段 内衰减率增加 $20 \mathrm{~dB} / \mathrm{m}$ 时, 车内噪声总值仅降低了 约 $0.1 \mathrm{~dB}(\mathrm{~A})$; 而当 $200 \sim 2500 \mathrm{~Hz}$ 频率段内衰减率 增加 $0.1 \mathrm{~dB} / \mathrm{m}$, 车内噪声降低约 $2.2 \mathrm{~dB}(\mathrm{~A})$, 当衰 减率提高 $0.3 \mathrm{~dB} / \mathrm{m}$ 时, 车内噪声降低约 $3.9 \mathrm{~dB}(\mathrm{~A})$, 此时车内噪声总值为 $79.4 \mathrm{~dB}(\mathrm{~A})$, 已低于司机室车 内噪声限值。随着衰减率的进一步提高, 车内噪声 将进一步降低, 但降低率逐渐减小。

(3) 提出了基于车内噪声的轨道衰减率控制研 究方法, 以轮轨噪声作为桥梁, 建立了车内噪声与 轨道衰减率之间的关系, 并得出了基于车内噪声的 轨道衰减率限值。

\section{参 考 文 献}

[1] THOMPSON D J. Wheel-rail noise generation, part IV : Contact zone and results[J]. Journal of Sound \& Vibration, 1993, 161(3): 447-466.

[2] REMINGTON P J. WHEEL/RAIL Noise-part 1 : Characterization of the wheel/rail dynamic system[J]. Journal of Sound \& Vibration, 1976, 46(3): 359-379.

[3] SIMONIWNICKI. Handbook of railway vehicle dynamics[M]. Boca Raton: CRC/Taylor \& Francis, 2006.

[4] THOMPSON D J. Experimental validation of the TWINS prediction program for rolling noise, part 2: Results[J]. Journal of Sound \& Vibration, 1996, 193(1): 123-135.

[5] THOMPSON D J, JONES C J C. A review of the modelling of wheel/rail noise generation[J]. Journal of Sound \& Vibration, 2000, 231(3): 519-536.

[6] THOMPSON D J. Experimental analysis of wave propagation in railway tracks[J]. Journal of Sound \& Vibration, 1997, 203(5): 867-888.

[7] JONES C J C, THOMPSON D J, DIEHL R J. The use of decay rates to analyse the performance of railway track in rolling noise generation[J]. Journal of Sound and Vibration, 2006, 293(3-5): 485-495.

[8] WU T X. On the railway track dynamics with rail vibration absorber for noise reduction[J]. Journal of Sound \& Vibration, 2008，309(3): 739-755.

[9] 孙晓静, 张厚贵, 刘维宁, 等. 轨道系统钢轨振动衰减 率动力测试研究[J]. 铁道工程学报, 2015, 32(7): 34-39. SUN Xiaojing, ZHANG Hougui, LIU Weining, et al. Test research on the characterizing the dynamic damping behavior of track structure[J]. Journal of Railway Engineering Society, 2015, 32(7): 34-39.

[10] ZHANG H, JIANG L, LI Y J, et al. Experimental study on controlling rail corrugation by tuning rail damper[J]. Applied Mechanics \& Materials, 2014, 587-589 : 1002-1006.

[11] TOWARD M, THOMPSON D J. Laboratory methods for testing the performance of acoustic rail dampers[C]// Nantes: Proceedings of the Acoustics, 2012: 3739-3744.

[12] 刘晓龙, 周信, 刘玉霞, 等. 约束型阻尼钢轨衰减率和 降噪效果试验分析 $[\mathrm{J}]$. 噪声与振动控制, 2015, 35(3): 24-28.

LIU Xiaolong, ZHOU Xin, LIU Yuxia, et al. Laboratory measurement of damped rail decay rates and analysis of noise reduction[J]. Noise and Vibration Control, 2015, 35(3): 24-28.

[13] Comite Europeen de Normalisation. BS EN 15461: 2008+A1: 2010. Railway applications- noise emission-characterisation of the dynamic properties of track sections for pass by noise measurements[S]. London : BSI-British Standards Institution, 2010.

[14] HAN J, XIAO X, WU Y, et al. Effect of rail corrugation on metro interior noise and its control[J]. Applied Acoustics, 2018, 130: 63-70.

[15] WU T W. Boundary element acoustics[M]. Southampton: WIT Press, 2005.

[16] ISO 3095: 2013. Institution B S. Acoustics-railway applications-measurement of noise emitted by railbound vehicles[S]. London: BSI-British Standards Institution, 2013.

[17] VERHEIJEN E, VAN BEEK A. Definition of track influence: Roughness in rolling noise[R]. Harmonoise Report Edited by AEAT-BV, 2003. 
[18] 中华人民共和国国家质量监督检验检疫总局, 中国国家标 准化管理委员会. GB 14892-2006 城市轨道交通列车噪 声限值和测量方法[S]. 北京: 中国标准出版社, 2006.

General Administration of Quality Supervision, Inspection and Quarantine of the People's Republic of China, Standardization Administration of the People's Republic of China. GB 14892 - 2006 Noise limit and measurement for train of urban rai transit[S]. Beijing: Standards Press of China, 2006

作者简介: 刘晓龙, 男, 1990 年出生, 博士研究生。主要研究方向为轨 道交通减振降噪。

E-mail: 1x1@my.swjtu.edu.cn

肖新标(通信作者), 男, 1978 年出生, 博士, 副研究员, 硕士研究生导 师。主要研究方向为轨道交通列车动力学, 轨道交通减振降噪。

E-mail: xinbiaoxiao@163.com 